\title{
BMJ Open Influence of the month of birth on persistence of ADHD in prospective studies: protocol for an individual patient data meta-analysis
}

\author{
Corentin J Gosling (D) , , ${ }^{1,2}$ Charlotte Pinabiaux, ${ }^{1}$ Serge Caparos, ${ }^{3,4}$ \\ Richard Delorme, ${ }^{5,6}$ Samuele Cortese $7,8,9,10,11$
}

To cite: Gosling CJ, Pinabiaux C, Caparos S, et al. Influence of the month of birth on persistence of $A D H D$ in prospective studies: protocol for an individual patient data meta-analysis. BMJ Open 2020;10:e040952. doi:10.1136/ bmjopen-2020-040952

- Prepublication history and supplemental material for this paper are available online. To view these files, please visit the journal online (http://dx.doi. org/10.1136/bmjopen-2020040952).

Received 27 May 2020 Revised 06 September 2020 Accepted 14 0ctober 2020

\section{A) Check for updates}

\section{(c) Author(s) (or their} employer(s)) 2020. Re-use permitted under CC BY-NC. No commercial re-use. See rights and permissions. Published by BMJ.

For numbered affiliations see end of article.

\section{Correspondence to}

Dr Corentin J Gosling; corentin.gosling@parisnanterre. $\mathrm{fr}$

\section{ABSTRACT}

Introduction Attention-deficit/hyperactivity disorder (ADHD) is a neurodevelopmental disorder with symptoms, especially the hyperactive ones, that tend to decrease in severity with age. Interestingly, children born just before the school-entry cut-off date (ie, the youngest pupils of a classroom) are at higher risk of being diagnosed with ADHD compared with children born just after the cutoff date. Noteworthy, this month-of-birth effect tends to disappear with increasing absolute age. Therefore, it is possible that young children erroneously diagnosed with ADHD due to their month of birth present a lower chance to have their diagnosis confirmed at a later age, artificially reinforcing the low persistence of ADHD across the lifespan. This protocol outlines an individual patient data (IPD) meta-analysis of prospective observational studies to explore the role of the month of birth in the low persistence of ADHD across the lifespan.

Methods and analysis Five databases will be systematically searched in order to find prospective observational studies where the presence of ADHD is assessed both at baseline and at a follow-up of at least 4 years. We will use a two-stage IPD meta-analytic approach to estimate the role of the month of birth in the persistence of ADHD. Various sensitivity analyses will be performed to assess the robustness of the results.

Ethics and dissemination No additional data will be collected and no de-identified raw data will be used. Ethics approval is thus not required for the present study. Results of this IPD meta-analysis will be submitted for publication in a peer-reviewed journal.

PROSPERO registration number CRD42020212650

\section{INTRODUCTION}

Attention-deficit/hyperactivity disorder (ADHD) is a neurodevelopmental disorder characterised by impairing and developmentally inappropriate levels of attention and/ or hyperactivity/impulsivity. ${ }^{1}$ Contrary to other neurodevelopmental disorders that have a relatively stable course across the lifespan, ${ }^{2-5}$ ADHD symptoms-at least those of the hyperactive domain-tend to fade with increasing age. A review of follow-up
Strength and limitations of this study

- A systematic review of prospective observational studies assessing the persistence of attentiondeficit/hyperactivity disorder (ADHD) will be performed.

- Based on studies retrieved in the systematic review, we will conduct the first individual patient data (IPD) meta-analysis assessing the role of the month of birth in the persistence of ADHD.

- Limitations of this study include both the potential difficulty in acquiring some IPD and the potential heterogeneity in ADHD diagnosis procedures.

studies assessing the long-term stability of ADHD diagnoses revealed that only one in six children with ADHD continue to meet the full criteria for ADHD into adulthood, even though impairing symptoms not necessarily meeting the threshold persist in about $70 \%$ of the childhood cases. ${ }^{6}$ Given the widespread burden caused by ADHD on adults' quality of life, ${ }^{7}$ identifying the mechanisms underlying the persistence of ADHD through the lifespan remains one of the main issues in the field.

Along with its particular developmental course, another peculiarity of ADHD lies in its close association with birth date. ${ }^{8}$ As shown in a very comprehensive study, including approximately one million participants recruited over a period of 11 years, children born in the last months of the civil year have a higher risk of being diagnosed with ADHD compared with children born in the first months of the year (this increase in risk is substantial, of about $40 \%) .{ }^{10}$ Since the school-entry cut-off date is the December 31 in most countries (ie, all children born between January 1 and December 31 start school the same year), children born at the end of the civil year may present with a higher 
level of cognitive and behavioural immaturity relative to their older classmates. This relative developmental bias could mimic some of the ADHD symptoms and could lead to inappropriate ADHD diagnoses in the youngest children. This interpretation has been reinforced by results showing that the month-of-birth effect can be reversed in some places. Children born in the last months of the civil year have a lower chance to be diagnosed with ADHD in regions where the school-entry cut-off date is in late August (ie, where children born from September to December are the oldest pupils in the classroom). ${ }^{11}$

Several studies have revealed that a key moderator of this month-of-birth effect on ADHD diagnosis is the absolute age of the children. As the age of the children increases, most studies show that the impact of the month of birth on ADHD diagnosis tends to decrease. ${ }^{12}$ To our knowledge, the mediator of this effect has not been explained empirically. Nevertheless, a common interpretation proposes that the effect of absolute age is most likely caused by the fact that a developmental difference of up to 12 months results in a more pronounced relative immaturity in young children than in adolescents or adults. ${ }^{13}$

In summary, compared with other neurodevelopmental disorders, ADHD presents two critical features: its developmental course, with severity and number of symptoms decreasing through the lifespan, and its high sensitivity to the month of birth, which gradually decreases with age. Taken together, these findings suggest that inappropriate diagnoses of ADHD due to the month of birth may contribute to the apparent low persistence of ADHD throughout the lifespan. Indeed, if the relative immaturity of a young child leads to an inappropriate diagnosis of ADHD, a reassessment of the original diagnosis several years later will most likely be associated with a reduction in ADHD symptoms. The aim of the present study will be to quantify the role of the month of birth as a possible factor contributing to the low persistence of ADHD throughout the lifespan. To this end, we will perform a systematic review of prospective observational studies assessing the persistence of ADHD with increased age and, through an individual patient data (IPD) meta-analysis, we will quantify the magnitude of the month-of-birth effect on the persistence of ADHD.

\section{METHOD}

The present IPD meta-analysis will be conducted in accordance with standard methods for IPD meta-analysis and reporting will conform to the Preferred Reporting Items for Systematic review and Meta-Analysis-IPD checklist (see the online supplemental table 1). ${ }^{1415}$

\section{Patient and public involvement}

There have been no patient and/or public involvement in the design of this IPD meta-analysis.

\section{Eligibility criteria}

Study design we will consider only prospective studies in which ADHD was diagnosed both at baseline and follow-up. A mean follow-up duration of 4 years after the initial diagnosis will be required (this criterion also applies to both the study level and the participant level).

Participants: we will consider children with either a categorical diagnosis of ADHD in accordance with Diagnostic and Statistical Manual of mental disorders, third edition (DSM-III), DSM-III-R, DSM-IV, DSM-IV-TR and DSM-5, or a categorical diagnosis of the hyperkinetic disorder in accordance with International Classification of Diseases, ninth edition (ICD-9) or ICD-10. This diagnosis should have been established using either a clinical interview or a validated questionnaire. Moreover, the participants should have received the initial (baseline) diagnosis before the age of 10 and the final (follow-up) diagnosis after the age of 10 . Studies in which ADHD is a comorbid, disorder secondary to a genetic syndrome will be excluded. Moreover, only participants living in a country/region in which a school-entry cut-off date is applied (eg, a country in which all children born between January 1 and December 31 of the same civil year start school during the same school year) will be included in our main analyses. Participants living in a country/region with no school-entry cut-off date will be included in a moderation analysis.

Outcome: the primary and only study outcome of the present study will be a categorical diagnosis of ADHD, consistent with standard classifications (as defined above). This diagnosis at follow-up should be performed at least 4 years after the diagnosis at baseline and should have occurred after the child has reached the age of 10 .

\section{Search methods for identification of studies}

In order to identify relevant studies, we will search five main databases (MEDLINE, Embase, CINAHL, PsycInfo and PubPsych). The search will be from inception up to September 2020. We will use controlled vocabulary (when available) and free text to search for two constructs, namely 'ADHD' and 'prospective studies' (see the online supplemental text 1). No date, publication type or language restriction will be applied.

All reference lists of included studies and relevant reviews will be screened to identify potentially eligible studies not found by the electronic searches. Moreover, authors of all included studies will be contacted to ask for published or unpublished data that could have been missed (see the Data collection and transfer section for details on the contact procedure).

\section{Data extraction}

Two review authors will independently screen the titles and abstracts to identify potentially eligible studies from the results of the searches. All disagreements will be resolved by discussion or, if impossible, the article will be processed to the next stage. Full texts of all reports selected on the basis of their title and/or abstract will be obtained and will be assessed against our inclusion criteria. Again, disagreements will be discussed and a senior author will be consulted if an agreement is not 
reached. All studies excluded after full-text reading will be recorded, along with the reason for their exclusion, in the online supplemental materials.

Two review authors will independently extract the following study-level data: name of first author and year of publication, publication type, name of the cohort, country in which the study took place, school-entry cut-off date, participation rate, percentage of attrition, sampling method (eg, cluster random sampling), mean follow-up duration, number of participants, mean age at baseline and at follow-up, diagnostic procedure used at baseline and at follow-up, diagnosis classification used at baseline and at follow-up, comorbidities. Authors of included studies will then be invited to confirm the accuracy of these data and then to access to the following participantlevel data: month of birth, diagnosis status at follow-up, age at baseline and follow-up, follow-up duration, psychiatric comorbidity, diagnosis procedure used at baseline and follow-up, and full-scale intelligence quotient.

If the school-entry cut-off date cannot be obtained by the authors of primary studies, we will contact the governmental administration of the country in which the study took place to obtain this cut-off date. Then, all months of birth will be recoded depending on the school-entry cut-off date (ie, month 1 will indicate the first month after the school-entry cut-off date). For example, a child born in January will be coded as born in month 1 if born in a region where the school-entry cut-off date is December 31 , but will be coded as born in month 5 if born in a region where the school entry cut-off date is August 31. The participants in studies conducted in a region where no strict school-entry cut-off date is applied will be coded with a 'non-applicable' month of birth and will be analysed separately.

\section{Risk of bias}

The risk of bias of the included studies will be assessed based on the Newcastle Ottawa Scale-cohort studies by two reviewers. ${ }^{16}$

\section{Data collection and transfer}

Because we anticipate that authors of included studies have not routinely reported the association between the month of birth and the persistence of ADHD, we plan to contact the authors of each included study. Corresponding authors of these included studies will be invited to collaborate with our team by e-mail. A maximum of two reminders will be sent. If we do not achieve to contact a corresponding author, the same procedure will be applied for the first and/or last author, if different from the corresponding author.

The month of birth is a possible de-identifying information and the diagnosis status is sensible data. Therefore, it is likely that participants of the included studies had not given their approval for sharing this information. We believe that requesting raw data would increase the likelihood that study authors may decline the invitation to participate in our project, increasing the bias in our final analyses. Therefore, authors will be invited to perform the analyses on their raw data and to share only the results of these analyses. The authors will be sent an $\mathrm{R}$ code to obtain the results automatically and homogeneously across studies.

\section{Statistical analysis}

All statistical analyses will be performed in $\mathrm{R}$ environment. No quantitative analysis will be performed for individual studies including less than 10 participants (and no sensitivity analysis will be performed for conditions including less than 10 participants). A meta-analysis will be performed for synthesising data from, at least, five studies. ${ }^{17}$ If data are acquired for less than five studies, they will be described qualitatively. No moderation analysis or publication bias analysis will be performed for less than 10 studies. ${ }^{18}$ We anticipate that a number of studies will meet inclusion criteria but will not be included in quantitative analysis (eg, because the month of birth will not be recorded, because we will not be able to obtain a response from study authors because the sample size was too small and so on). Characteristics of eligible studies included in the quantitative analysis will be compared with those not included in quantitative analysis.

We will perform an IPD meta-analysis using only the two-stage approach. ${ }^{19}$ The choice to rely on the two-stage approach is based on a number of considerations. First, as stated earlier, we believe that requesting to share raw data (which is needed in the one-stage approach but not in the two-stage) may decrease the rate of data inclusion. Second, a major drawback of the one-stage approach lies in its convergence issues, which can be reinforced by the use of a fully specified model and a binary dependent variable (ADHD diagnosis at follow-up confirmed or not confirmed).$^{20}$ Taken together, and because one-stage and two-stage approaches generally produce similar results, ${ }^{21}$ we chose to rely only on the two-stage approach.

For each study, we will fit a logistic regression model assessing the linear effect of month of birth (predictor) on the persistence of ADHD at follow-up (dependent variable) to obtain the log odds ratio (OR). Any participant with a missing month of birth or ADHD diagnosis at follow-up will be excluded from this main analysis. Then, the pooled estimate will be obtained by random-effects meta-analysis (using the restricted maximum likelihood estimator in 'metafor' package in R). Heterogeneity will be assessed using Cochran's $Q$ and $I^{2}$ statistics. The heterogeneity source and robustness of our findings will be assessed in several sensitivity and moderation analyses. In the sensitivity analyses, we will reassess our primary analysis (1) on participants with a follow-up duration superior to 10 years; (2) on participants with a baseline diagnosis performed before the age of 8 and a follow-up diagnosis performed after the age of 16 ; (3) on participants with identical diagnosis procedure at baseline and at follow-up; (4) on participants with no comorbid psychiatric disorder; (5) on studies with a low percentage of attrition (inferior or equal to 20\%); (6) when effect sizes 
of individual studies are obtained using a robust logistic regression (using the 'robustbase' package in $\mathrm{R}$ ) ${ }^{22}$; (7) when influential studies (identified using Cook's distance) are removed (using the 'metafor' package in $\mathrm{R})^{23}$ and (8) when missing values are handled using multiple imputations (using the 'mice' package in R) ${ }^{24}$ For imputation models, we will fit a model with no auxiliary variables and a model with all recorded variables as auxiliary variables. In the moderation analyses, we will investigate the role of two study-level moderators and one patient-level moderator. For study-level moderation analysis, we will start by investigating the influence of the presence of a schoolentry cut-off in the region/country in which primary studies took place on the association between the month of birth and ADHD persistence. This moderator is thus a study-level binary moderator (presence vs absence of a school-entry cut-off). Then, we will explore the influence of the diagnosis procedure (questionnaires vs clinical interview) on the association between the month of birth and ADHD persistence. This moderator is also a studylevel binary moderator. For the patient-level moderation analysis, we will explore the influence of ADHD subtypes (or presentation, as per DSM-5) on the association of month of birth with ADHD persistence. In order to do so, we will assess the influence of the month of birth on ADHD persistence for each ADHD subtypes/presentation in individual studies. Then, a moderation analysis and an average estimate effect for each subtype/presentation will be obtained using a two-stage meta-analysis without model intercept. Last, publication bias will be detected by a $\mathrm{p}$ value of the Egger test inferior to 0.10 .

\section{Ethics and dissemination}

No raw data will be transferred for this IPD meta-analysis and thus does not require any supplemental ethics committee approval-as any standard meta-analysis. The findings of this study are planned to be disseminated through peer-reviewed publications and/or conference presentations.

\section{Author affiliations}

${ }^{1}$ Department of Psychology, DysCo Lab, Paris-Nanterre University, Nanterre, France ${ }^{2}$ Department of Psychology, EA 4057, Université de Paris, Paris, France

${ }^{3}$ Department of Psychology, DysCo Lab, Paris 8 University, Saint-Denis, France

${ }^{4}$ Institut Universitaire de France, Paris, France

${ }^{5}$ Department of Child and Adolescent Psychiatry, Assistance Publique - Hopitaux de Paris, Paris, France

${ }^{6}$ Human Genetics and Cognitive Functions, Institut Pasteur, Paris, France

${ }^{7}$ Center for Innovation in Mental Health, School of Psychology, Faculty of

Environmental and Life Sciences, University of Southampton, Southampton, UK

${ }^{8}$ Clinical and Experimental Sciences (CNS and Psychiatry), Faculty of Medicine, University of Southampton, Southampton, UK

${ }^{9}$ Solent NHS Trust, Southampton, UK

${ }^{10}$ Hassenfeld Children's Hospital at NYU Langone, New York University Child Study Center, New York City, New York, USA

${ }^{11}$ Division of Psychiatry and Applied Psychology, School of Medicine, University of Nottingham, Nottingham, UK

Twitter Samuele Cortese @CorteseSamuele

Contributors CJG, CP, SCa, RD and SCo have designed research. CJG, CP, SCa, RD and $\mathrm{SCo}$ have either written or edited the manuscript.
Funding The authors have not declared a specific grant for this research from any funding agency in the public, commercial or not-for-profit sectors.

Competing interests SCo declares honoraria and reimbursement for travel and accommodation expenses for lectures from the following non-profit associations: Association for Child and Adolescent Central Health (ACAMH), Canadian ADHD Alliance Resource (CADDRA), British Association of Pharmacology (BAP), and from Healthcare Convention for educational activity on ADHD. All other authors have no conflict of interest to declare.

Patient consent for publication Not required.

Provenance and peer review Not commissioned; externally peer reviewed.

Supplemental material This content has been supplied by the author(s). It has not been vetted by BMJ Publishing Group Limited (BMJ) and may not have been peer-reviewed. Any opinions or recommendations discussed are solely those of the author(s) and are not endorsed by BMJ. BMJ disclaims all liability and responsibility arising from any reliance placed on the content. Where the content includes any translated material, BMJ does not warrant the accuracy and reliability of the translations (including but not limited to local regulations, clinical guidelines, terminology, drug names and drug dosages), and is not responsible for any error and/or omissions arising from translation and adaptation or otherwise.

Open access This is an open access article distributed in accordance with the Creative Commons Attribution Non Commercial (CC BY-NC 4.0) license, which permits others to distribute, remix, adapt, build upon this work non-commercially, and license their derivative works on different terms, provided the original work is properly cited, appropriate credit is given, any changes made indicated, and the use is non-commercial. See: http://creativecommons.org/licenses/by-nc/4.0/.

ORCID iD

Corentin J Gosling http://orcid.org/0000-0003-1133-9344

\section{REFERENCES}

1 American Psychiatric Association. Diagnostic and statistical manual of mental disorders. 5th ed. Arlington, VA: Author, 2013.

2 Pierce K, Gazestani VH, Bacon E, et al. Evaluation of the diagnostic stability of the early autism spectrum disorder phenotype in the general population starting at 12 months. JAMA Pediatr 2019;173:578-87.

3 Peterson RL, Pennington BF, Olson RK, et al. Longitudinal stability of phonological and surface subtypes of developmental dyslexia. Sci Stud Read 2014;18:347-62.

4 Shaywitz SE, Fletcher JM, Holahan JM, et al. Persistence of dyslexia: the Connecticut longitudinal study at adolescence. Pediatrics 1999;104:1351-9.

5 Woolfenden S, Sarkozy V, Ridley G, et al. A systematic review of the diagnostic stability of autism spectrum disorder. Res Autism Spectr Disord 2012;6:345-54.

6 Faraone SV, Biederman J, Mick E. The age-dependent decline of attention deficit hyperactivity disorder: a meta-analysis of follow-up studies. Psychol Med 2006;36:159-65.

7 Barkley RA. Attention-Deficit hyperactivity disorder: a Handbook for diagnosis and treatment. 4edn. New York: Guilford, 2015.

8 Elder TE. The importance of relative standards in ADHD diagnoses: evidence based on exact birth dates. J Health Econ 2010;29:641-56.

9 Evans WN, Morrill MS, Parente ST. Measuring inappropriate medical diagnosis and treatment in survey data: the case of ADHD among school-age children. J Health Econ 2010;29:657-73.

10 Morrow RL, Garland EJ, Wright JM, et al. Influence of relative age on diagnosis and treatment of attention-deficit/hyperactivity disorder in children. CMAJ 2012;184:755-62.

11 Sayal K, Prasad V, Daley D, et al. Adhd in children and young people: prevalence, care pathways, and service provision. Lancet Psychiatry 2018;5:175-86.

12 Hoshen MB, Benis A, Keyes KM, et al. Stimulant use for ADHD and relative age in class among children in Israel. Pharmacoepidemiol Drug Saf 2016;25:652-60.

13 Whitely M, Raven M, Timimi S, et al. Attention deficit hyperactivity disorder late birthdate effect common in both high and low prescribing international jurisdictions: a systematic review. J Child Psychol Psychiatry 2019;60:380-91.

14 Riley RD, Lambert PC, Abo-Zaid G. Meta-Analysis of individual participant data: rationale, conduct, and reporting. BMJ 2010;340:c221.

15 Stewart LA, Clarke M, Rovers M, et al. Preferred reporting items for systematic review and meta-analyses of individual participant data: the PRISMA-IPD statement. JAMA 2015;313:1657-65. 
16 Wells GA, Shea B, O'Connell D, et al. The Newcastle-Ottawa scale (NOS) for assessing the quality if nonrandomized studies in metaanalyses, 2009. Available: http://www.ohri.ca/programs/clinical_ epidemiology/oxford.htm [Accessed 2009 Oct 19].

17 Jackson D, Turner R. Power analysis for random-effects metaanalysis. Res Synth Methods 2017;8:290-302.

18 Higgins JPT, Green S. Cochrane Handbook for systematic reviews of interventions version 5.1.0, 2011. Available: www.handbook. cochrane.org

19 Burke DL, Ensor J, Riley RD. Meta-Analysis using individual participant data: one-stage and two-stage approaches, and why they may differ. Stat Med 2017;36:855-75.
20 Kontopantelis E. A comparison of one-stage vs two-stage individual patient data meta-analysis methods: a simulation study. Res Synth Methods 2018;9:417-30.

21 Debray TPA, Riley RD, Rovers MM, et al. Individual participant data (IPD) meta-analyses of diagnostic and prognostic modeling studies: guidance on their use. PLoS Med 2015;12:10.

22 Maechler M, Rousseeuw P, Croux C, et al. robustbase: basic robust statistics, 2020. Available: http://robustbase.r-forge.r-project.org/

23 Viechtbauer W. Conducting Meta-Analyses in $R$ with the metafor Package. J Stat Softw 2010;36:1-48.

24 van Buuren S, Groothuis-Oudshoorn K. "mice: Multivariate Imputation by Chained Equations in R.". Journal of Statistical Software 2011;45:1-67. 